\title{
Predicting the population dynamics in Amoebophrya parasitoids and their dinoflagellate hosts using a mathematical model
}

\author{
Paulo S. Salomon ${ }^{1, *}$, Willem Stolte ${ }^{1,2}$ \\ ${ }^{1}$ Marine Sciences Centre, Linnaeus University, 39182, Kalmar, Sweden \\ ${ }^{2}$ Present address: Deltares, Postbus 177, 2600 MH Delft, The Netherlands
}

ABSTRACT: Free-living, photosynthetic marine dinoflagellates are frequently infected by microparasites of the genus Amoebophrya. Attacks by Amoebophrya can contribute to the termination of dinoflagellate blooms and have been suggested to influence the geographical distribution of certain host species. Because infection terminates with the killing of the host (i.e. Amoebophrya behaves like a parasitoid), the interaction can be considered, from a modeling point of view, similar to the process of predation, with the difference that it takes a longer time for the parasitoid to kill the host as compared to typical predator-prey interactions. In the present work, we explored the population dynamics in Amoebophrya and their dinoflagellate hosts using the Rosenzweig-MacArthur modification of the traditional Lotka-Volterra predation model. The model was parameterized for 3 systems, Akashiwo sanguinea, Gymnodinium instriatum, and Karlodinium micrum, and their respective Amoebophrya parasitoids, using published experimental data. Parameter validation was possible for parasitoid search rate and mortality. The potential for host control by Amoebophrya and the probability for host extinction were studied with respect to carrying capacity, a parameter that is influenced by e.g. eutrophication. The model may be useful to predict conditions under which Amoebophrya can control populations of its dinoflagellate hosts.

KEY WORDS: Interspecific interactions · Parasitism · Marine dinoflagellates · Mathematical model Amoebophrya

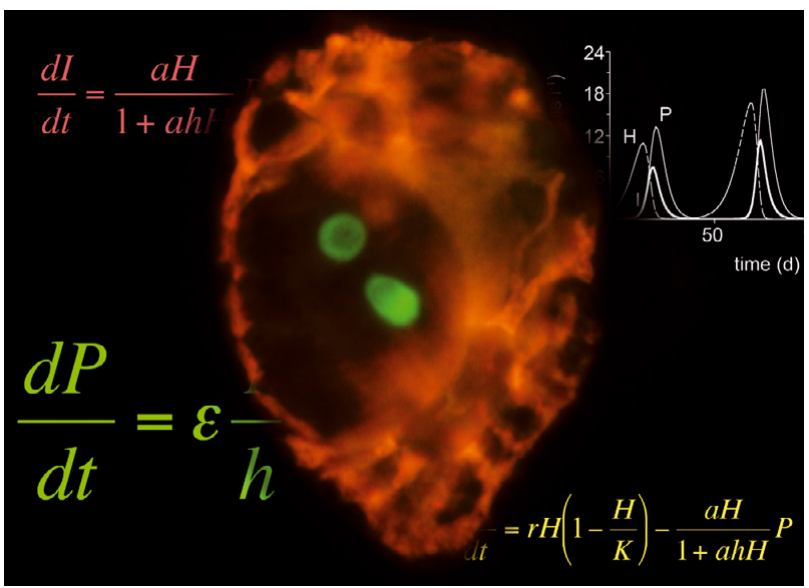

Marine dinoflagellate Dinophysis norvegica infected with Amoebophrya sp. Orange: host's pigments; green: earlystage Amoebophrya revealed by FITC-labelled FISH probes.

Photo and image: Paulo S. Salomon

\section{INTRODUCTION}

The parasitic dinoflagellate genus Amoebophrya is a widespread member of marine plankton communities. One group of such parasitoids, initially described as 1 species, A. ceratti (Koeppen) Cachon, infects more than 40 different free-living dinoflagellate species from 23 genera in coastal waters worldwide (Salomon et al. 2003a, 2009, Park et al. 2004). The Amoebophrya life cycle alternates between an extra- and an intracellular phase. Infection is mediated by free-living, small flagellated forms (typically $<10 \mu \mathrm{m}$ ) called dinospores. Infected hosts serve as a nutrient source for the tro- 
phont, which grows into a multinucleated stage (syncytium) that emerges from the host cell as a vermiform (Cachon \& Cachon 1987). The duration of the intracellular phase for cultured host-Amoebophrya systems ranges from ca. 1.6 to $3 \mathrm{~d}$, and infection always seems to lead to death of the host cell (Coats \& Bockstahler 1994, Coats \& Park 2002, Kim et al. 2004). Once emerged, the vermiform undergoes cytokinesis to produce numerous (typically 50 to 500) dinospores, completing the cycle (Coats \& Park 2002). Several dinospores can attack and penetrate a single host cell, but normally only 1 matures (Coats \& Park 2002, Salomon et al. 2003a, 2006).

Host-specificity of Amoebophrya spp. infecting dinoflagellates has been debated in the literature. Observations of morphologically similar trophonts inside cooccurring hosts in natural dinoflagellate assemblages led to the idea of low specificity by the parasitoid (Nishitani et al. 1985). The further establishment of Amoebophrya in cultures together with their primary hosts allowed cross-infection experiments that indicated high host-specificity, challenging the previous idea (Coats et al. 1996, Coats \& Park 2002). Comparison of SSU rDNA sequences from Amoebophrya that originated from different dinoflagellates revealed high genetic diversity within this group, supporting the hypothesis of a species complex with some degree of host-specificity (Gunderson et al. 1999, Janson et al. 2000, Salomon et al. 2003a, b, Kim et al. 2008). However, a recent study, also using cultured systems, has shown that 2 Amoebophrya strains isolated from Alexandrium affine and Gonyaulax polygramma have wide host ranges (Kim 2006). The data accumulated hitherto indicate that infection by the $A$. ceratii species complex is restricted to the dinoflagellate group. However, high host-specificity is a characteristic of some, but not all, Amoebophrya spp.

Several dinoflagellate species that are suitable hosts for Amoebophrya are bloom forming and/or toxin producing and are harmful to the aquatic biota and also to humans via ingestion of contaminated seafood (Hallegraeff 2003). The ability of Amoebophrya to lethally infect harmful dinoflagellate species, high prevalence levels observed in nature, and the narrow host range displayed by several strains/species within the genus, has led to speculations for its use as a mitigating agent against the occurrence of harmful dinoflagellates (Taylor 1968, Coats et al. 1996, Anderson 1997, Chambouvet et al. 2008). To use it as an effective agent, 2 prerequisites are important. Firstly, the parasitoid should be selective enough so that no or few host species other than the target dinoflagellate is affected. Secondly, it must be able to control the growth and/or biomass of the target host species. Moreover, it is important to know the required concentration of parasitoid dino- spores to be released in a given area to have the desired effect. Also, Amoebophrya dinospores can be grazed by other components of pelagic microbial food webs, creating temporary, and at times significant, trophic links (Maranda 2001, Johansson \& Coats 2002). Understanding the population dynamics in parasitoids and hosts is thus vital to predict the effect of additions to control dinoflagellate populations, as well as their impact on the functioning of marine pelagic ecosystems.

Due to the limited possibilities to sample marine waters on small spatial and temporal scales, the population dynamics in Amoebophrya-dinoflagellate systems are largely unknown. In natural waters, Amoebophrya prevalence in dinoflagellate hosts can escalate up to $80 \%$ and higher during epidemics (Coats et al. 1996). However, most commonly reported infection prevalences are much lower than that (Coats \& Bockstahler 1994, Salomon et al. 2003a, 2006, 2009, Chambouvet et al. 2008). Thus far, the majority of the field work on Amoebophrya has focused on the intracellular phase of infection. Only recently, with the help of fluorescent probes, the abundance of free-living Amoebophrya stages has been reported for marine waters (Chambouvet et al. 2008).

Models of dynamics in a single species are usually hindered by the high number of possible interactions such as competition, grazing/predation, and infection by pathogens. Parasite-host systems, however, are often characterized by a relatively high specificity and are therefore more suitable to be modeled.

Host-parasite dynamics have been described through mathematical models mostly using the traditional Anderson-May formulation (May 1978, Anderson \& May 1980, 1981) that combines elements of traditional epidemiology with those of predator-prey systems, assuming linear relationships among the organisms involved. Recently, Montagnes et al. (2008) used such formulations, embedded in a food web model, to simulate the relative effects of grazers and Amoebophrya parasites on pelagic dinoflagellate populations. However, a pure predator-prey, Lotka-Volterra-like formulation also seems to be a valid approach to simulate the dynamics in Amoebophrya infecting dinoflagellates, because the infection terminates with the assassination of the host in a typical parasitoid behavior (Coats \& Bockstahler 1994, Coats \& Park 2002, Kim et al. 2004). For this reason, in the present work, the Rosenzweig-MacArthur modification of the traditional Lotka-Volterra predation model (Rosenzweig 1971) was chosen as the starting point for a model formulation to study the dynamics of Amoebophrya and its dinoflagellate hosts.

This study aimed to formulate and parameterize a model based on information acquired from Amoebophrya-dinoflagellate systems kept in culture. This 
model was used to formulate testable hypotheses on parasitoid-host dynamics in natural waters based on the following question: What are the impacts of Amoebophrya spp. on their hosts' biomass and the system dynamics at varying environmental carrying capacities (determined by e.g. nutrient levels)?

\section{MATERIALS AND METHODS}

Model formulation. Parasitoid behavior resembles that of a predator, i.e. the interaction ends with the 'assassination' of the host. Infective offspring are produced shortly after emergence of the vermiform from the host cell. Infection by a single parasitoid is presumably enough to kill a host. Based on these features, we chose to explore the system dynamics starting with the Rosenzweig-MacArthur modification of the traditional Lotka-Volterra predation model (Rosenzweig 1971), here named Model I

$$
\begin{gathered}
\frac{\mathrm{d} H}{\mathrm{~d} t}=r H\left(1-\frac{H}{K}\right)-\frac{a H}{1+a h H} P \\
\frac{\mathrm{d} P}{\mathrm{~d} t}=\frac{\varepsilon a H}{1+a h H} P-m P
\end{gathered}
$$

where $H$ and $P$ represent host and free-living parasitoid concentrations, respectively. Reproduction rate of the host is represented by a logistic function, with $r$ as the maximal reproduction rate (at $H=0$ ) and $K$ as the carrying capacity. The infection rate depends on the concentration of both parasitoid and host, and is modeled as Holling Type II dynamics of infection with search rate $a$ and handling time $h$ of the parasitoid for the host. The number of newly produced Amoebophrya dinospores per infected host is $\varepsilon$. Finally, $m$ is the natural mortality rate of the free-living dinospores.

This system of ordinary differential equations (ODEs) has been extensively analyzed, and the dynamics for each parameter value are known, as well as critical limits for stability and coexistence (Rosenzweig 1971). There is a critical carrying capacity $K_{\mathrm{C}}$ for the host defined by:

$$
K_{\mathrm{C}}=\frac{m}{a(\varepsilon-m h)}
$$

below which there is no stable state with parasitoids present. At $K>K_{\mathrm{C}}$, coexistence of both parasitoid and host is possible. Stable coexistence occurs for $K_{\mathrm{C}}<K<$ $K_{\mathrm{S}}$ where

$$
K_{\mathrm{S}}=\frac{\varepsilon+m h}{a h(\varepsilon-m h)}
$$

For $K>K_{\mathrm{S}}$, unstable coexistence occurs and both host and parasitoid populations will fluctuate similar to Lotka-Volterra oscillations.

A drawback of Model I is that the infected host stage is not explicitly modeled. This makes comparison between model outcomes and field data difficult, because Amoebophrya data in natural samples are almost exclusively in the form of percentage of infected hosts. To solve this problem, we made a modification similar to that described by May \& Anderson (1978), creating the state variable $I$ (number of infected hosts) and 1 extra differential equation to describe its dynamics (this modified model is named Model II):

$$
\begin{gathered}
\frac{\mathrm{d} H}{\mathrm{~d} t}=r H\left(1-\frac{H}{K}\right)-\frac{a H}{1+a h H} P \\
\frac{\mathrm{d} I}{\mathrm{~d} t}=\frac{a H}{1+a h H} P-\frac{I}{h} \\
\frac{\mathrm{d} P}{\mathrm{~d} t}=\varepsilon \frac{I}{h}-m P
\end{gathered}
$$

Parameterization. The model was parameterized for 3 dinoflagellate species (Akashiwo sanguinea, Gymnodinium instriatum, previously known as Gyrodinium instriatum and synonymous with Gyrodinium uncatenum, and Karlodinium micrum, currently known as $K$. venificum) and their respective Amoebophrya sp., using experimental data published by Coats \& Park (2002) (Table 1). Per capita growth rate $(r)$ of the hosts was calculated from the increase in host cell concentration in the initial phase of the experiment (prior to the detection of late stage Amoebophrya trophonts at a 1:1 dinospore:host ratio) presented in Fig. 4 of

Table 1. Parameter values for the 3 Amoebophrya-dinoflagellate systems modeled in the present study. Values were taken directly or calculated based on data from Coats \& Park (2002). A. san: Akashiwo sanguinea, G. ins: Gymnodinium instriatum, K. mic: Karlodinium micrum

\begin{tabular}{|lccccc|}
\hline \multirow{2}{*}{ Parameter } & Explanation & Unit & A. san & G. ins & K. mic \\
\hline$r$ & Host per capita growth rate & $\mathrm{d}^{-1}$ & 0.21 & 0.24 & 0.35 \\
$a$ & Parasitoid search rate & ldinospore $^{-1} \mathrm{~d}^{-1}$ & $4.40 \times 10^{-7}$ & $5.58 \times 10^{-8}$ & $1.34 \times 10^{-8}$ \\
$h$ & Parasitoid handling time & $\mathrm{d}$ & 2.96 & 2.79 & 2.46 \\
$\varepsilon$ & Parasitoid reproductive output & dinospores (killed host) $)^{-1}$ & 600 & 550 & 40 \\
$m$ & Parasitoid mortality rate & $\mathrm{d}^{-1}$ & 0.48 & 1.02 & 0.26 \\
\hline
\end{tabular}


Coats \& Park (2002) assuming exponential growth. Search rate (a) of the parasitoid for the host equals the volume of water that a dinospore is able to search for hosts per day. Search rate can be defined as the initial slope $(\alpha)$ of the relation between infection rate of the parasite versus host concentration. Data for calculation of search rates were found in Coats \& Park (2002), their Fig. 1. This figure shows plots for percentage of infected hosts after a given period of time versus initial parasite concentration, with time range variable for each host (36 h for A. sanguinea, $40 \mathrm{~h}$ for $G$. instriatum, and $35 \mathrm{~h}$ for $K$. micrum). The $\alpha$ values for each species were divided by 100 , because the value is given in percentage, and multiplied by 1000 parasites (1:1 ratio). This value was divided by 1000 parasites and 1000 hosts and $1000 \mathrm{ml}$ (to express the result in liters). To express search rate on a per day basis (i.e. 1 dinospore $^{-1} \mathrm{~d}^{-1}$ ), this last value was divided by the time (in hours) that each species was incubated, multiplied by 24:

$a=\frac{\alpha}{100 \%}$

$$
\times \frac{\left.10^{3} \text { (parasites }\right) \cdot 24(\mathrm{~h} / \text { day })}{\left.10^{3} \text { (parasites }\right) \cdot 10^{3}(\text { hosts }) \cdot 10^{3}(\mathrm{ml}) \cdot \text { incubation_time }(\mathrm{h})}
$$

where $\alpha$ is the initial slope (in percentage) between percentage of infected hosts versus dinospore inoculum sizes of Fig. 1 in Coats \& Park (2002). Handling time $(h)$ was assumed as the total generation time for the parasite (Coats \& Park 2002). Amoebophrya reproductive output $(\varepsilon)$ was estimated as the proportion between the maximum number of dinospores observed during the experiments and the maximum number of infected cells in the preceding period from Fig. 4 in Coats \& Park (2002). Mortality rate values of dinospores $(m)$ were taken directly from Coats \& Park (2002), their Fig. 2, calculated as the first-order decay constant of dinospore concentration versus time.

Numerical simulation. Models I and II were numerically integrated on a personal computer using SIMILE (www.simulistics.com) for simulations, and the ODESOLVE package available within the free software environment ' $\mathrm{R}$ ' (www.r-project.org/) was used for bifurcation analysis. Model files and scripts are available from the corresponding author.

Stability of the system. For Model I, values of $K_{\mathrm{C}}$ and $K_{\mathrm{S}}$ were obtained directly from Eqs. (3) and (4). For the formulation in Model II, the model was run for 1200 simulated days (maximum time step $0.1 \mathrm{~d}$ ) at varying values of $K$ to investigate the effect of $K$ on the stability of the system. If the system reached the stable equilibrium within the simulated time, the equilibrium value was plotted against $K$. Conversely, if the system was unstable, the maximum and minimum values of the oscillations were calculated, and maximum and mini- mum variable values within the last 200 simulation days were plotted against $K$. Values of $K_{\mathrm{C}}$ and $K_{\mathrm{S}}$ for Model II were obtained graphically from the resulting bifurcation diagrams $H$ and $P$ versus $K$.

Sensitivity analysis. The effect of parameter values on parasitoid prevalence was tested for Amoebophrya-

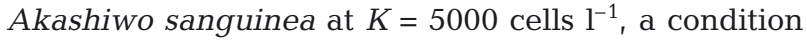
which allows for stable coexistence. All parameter values except $K$ were individually increased and decreased up to $25 \%$ of initial values (Table 1), and the resulting parasitoid prevalence deviation (in \%) from the originally parameterized model was plotted against the deviation for each parameter in $\%$.

\section{RESULTS}

\section{Comparison with experimental data}

To validate the estimated values of the parameters $a$ and $m$, Model II was run with initial conditions equal to experimental data in Coats \& Park (2002), their Fig. 4, and the results are compared in Fig. 1. The values of $\varepsilon, h$, and $r$ were estimated from the same experiments and for that reason, the model could only be validated with respect to $a$ and $m$. In general, the model outcome agreed reasonably well with the experimental data. For Akashiwo sanguinea, infection rate at the low dinospore:host ratio (1:1) was clearly overestimated by the model, resulting in far more modeled infected cells. The opposite was seen for Karlodinium micrum, where the high percentages of infected cells were not met by the model results at both dinospore:host ratios (1:1 and 40:1).

\section{System stability}

With our parameterization, the critical carrying capacity above which stable coexistence of host and parasitoid occur $\left(K_{\mathrm{C}}\right)$ would be $1.82 \times 10^{3}, 3.34 \times 10^{4}$ and $4.93 \times 10^{5}$ cells $\mathrm{l}^{-1}$ for Akashiwo sanguinea, Gymnodinium instriatum, and Karlodinium micrum, respectively, when applying Eqs. (3) and (4). The critical carrying capacity above which stable coexistence is changed to periodic cycles $\left(K_{\mathrm{S}}\right)$ was 2 to 3 orders of magnitude higher than $K_{\mathrm{C}}$ (Table 2). These results were in agreement with the numerically integrated simulations (data not shown).

Similar to Model I, Model II showed 3 different stability states depending on the value of $K$ (Fig. 2). $K_{\mathrm{C}}$ values for Model II, judged from the curves of cell concentration versus $K$ (Fig. 2), were very similar to $K_{\mathrm{C}}$ values for Model I. Overall, $K_{\mathrm{S}}$ for Model II was 13 to 86 times lower than $K_{\mathrm{S}}$ for Model I (Table 2). 

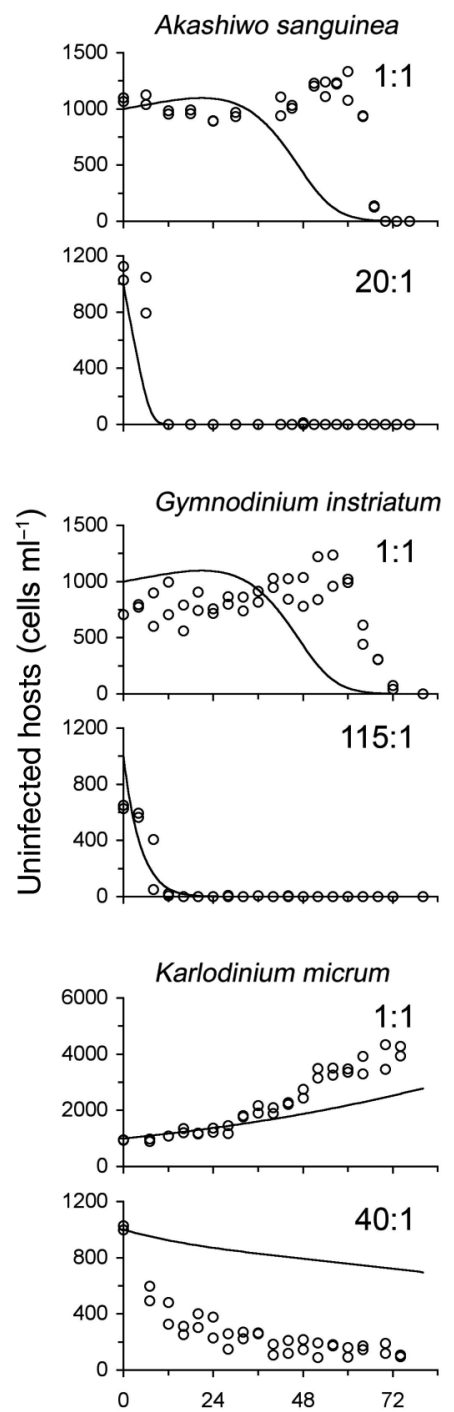
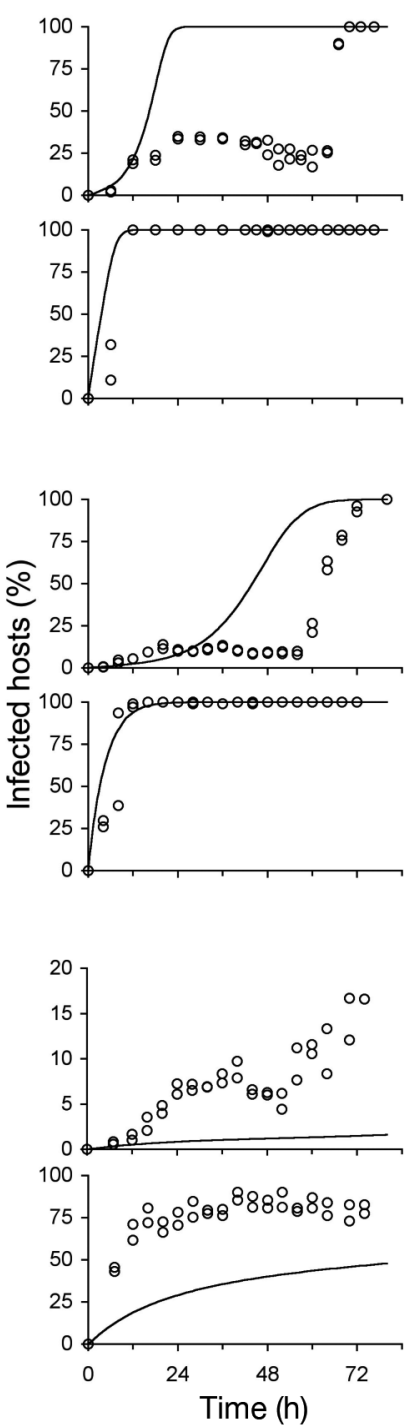

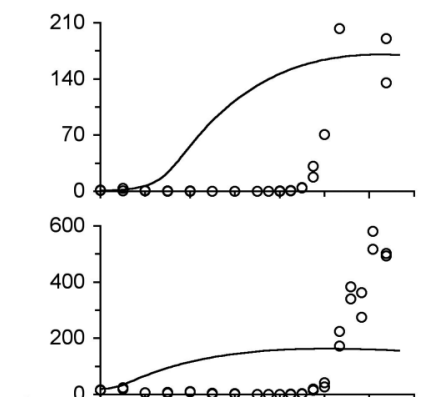

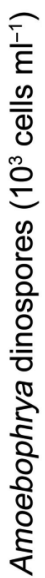

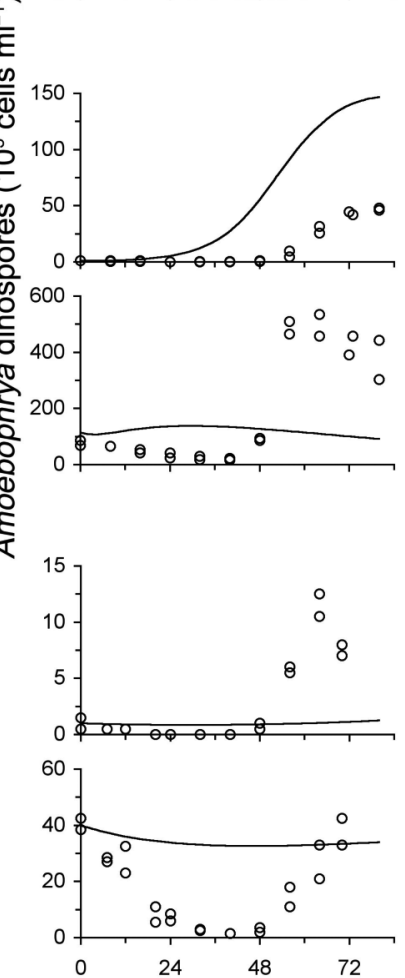

Fig. 1. Amoebophrya infecting Akashiwo sanguinea, Gymnodinium instriatum, and Karlodinium micrum. Model II simulations (solid lines) and experimental data (open circles) from Coats \& Park (2002). Parameter values were as in Table 1. The runs are a validation of the model with regard to Amoebophrya dinospore search rate $(a)$ and mortality rate $(m)$, which were estimated from independent experiments. $K$ was set to $1 \times 10^{7}$ cells $1^{-1}$ for $A$. sanguinea, $5 \times 10^{6}$ cells $^{-1}$ for $G$. instriatum, and $2 \times 10^{7}$ cells $\mathrm{l}^{-1}$ for

\section{K. micrum}

Table 2. Model I and II outputs regarding critical values for carrying capacity of the environment $(K)$, in cells $\mathrm{l}^{-1}$, for the 3 Amoebophrya-dinoflagellate systems used in this study. $K_{\mathrm{C}}$ : critical $K$ for stable coexistence (calculated from Eq. 3), $K_{\mathrm{S}}$ : critical $K$ for unstable coexistence (Model I: calculated from Eq. 4, Model II: estimated graphically from Fig. 2). A. san: Akashiwo sanguinea, G. ins: Gymnodinium instriatum, K. mic: Karlodinium micrum

\begin{tabular}{|lcccc|}
\hline Host & \multicolumn{2}{c}{ Model I } & Model II & \multicolumn{2}{c|}{$K_{\mathrm{S}}$ Model I: } \\
& $K_{\mathrm{C}}$ & $K_{\mathrm{S}}$ & $K_{\mathrm{S}}$ & $K_{\mathrm{S}}$ Model II \\
\hline A. san & $1.8 \times 10^{3}$ & $7.7 \times 10^{5}$ & $0.9 \times 10^{4}$ & 86 \\
G. ins & $3.3 \times 10^{4}$ & $6.5 \times 10^{6}$ & $1.7 \times 10^{5}$ & 38 \\
K. mic & $4.9 \times 10^{5}$ & $3.1 \times 10^{7}$ & $2.4 \times 10^{6}$ & 13 \\
\hline
\end{tabular}

\section{Temporal dynamics}

An example of the temporal dynamics using Model II is shown in Fig. 3 for the host Akashiwo sanguinea. At $K<K_{\mathrm{C}}$ parasitoids go extinct, and host concentrations increase up to $K$ (Fig. 3a). At $K_{\mathrm{C}}<K<K_{\mathrm{S}}$, peaks of host abundance and prevalence occur with intervals of ca. $6 \mathrm{wk}$ until stable coexistence, or, at relatively low $K$, stable coexistence is reached without marked oscillations (Fig. 3b). At $K>K_{\mathrm{S}}$, the initial time interval between peaks is similar to the previous case, increasing with time both in amplitude and frequency (Fig. 3c). A typical feature observed in the simulations of all 3 systems was long periods of time with very low 

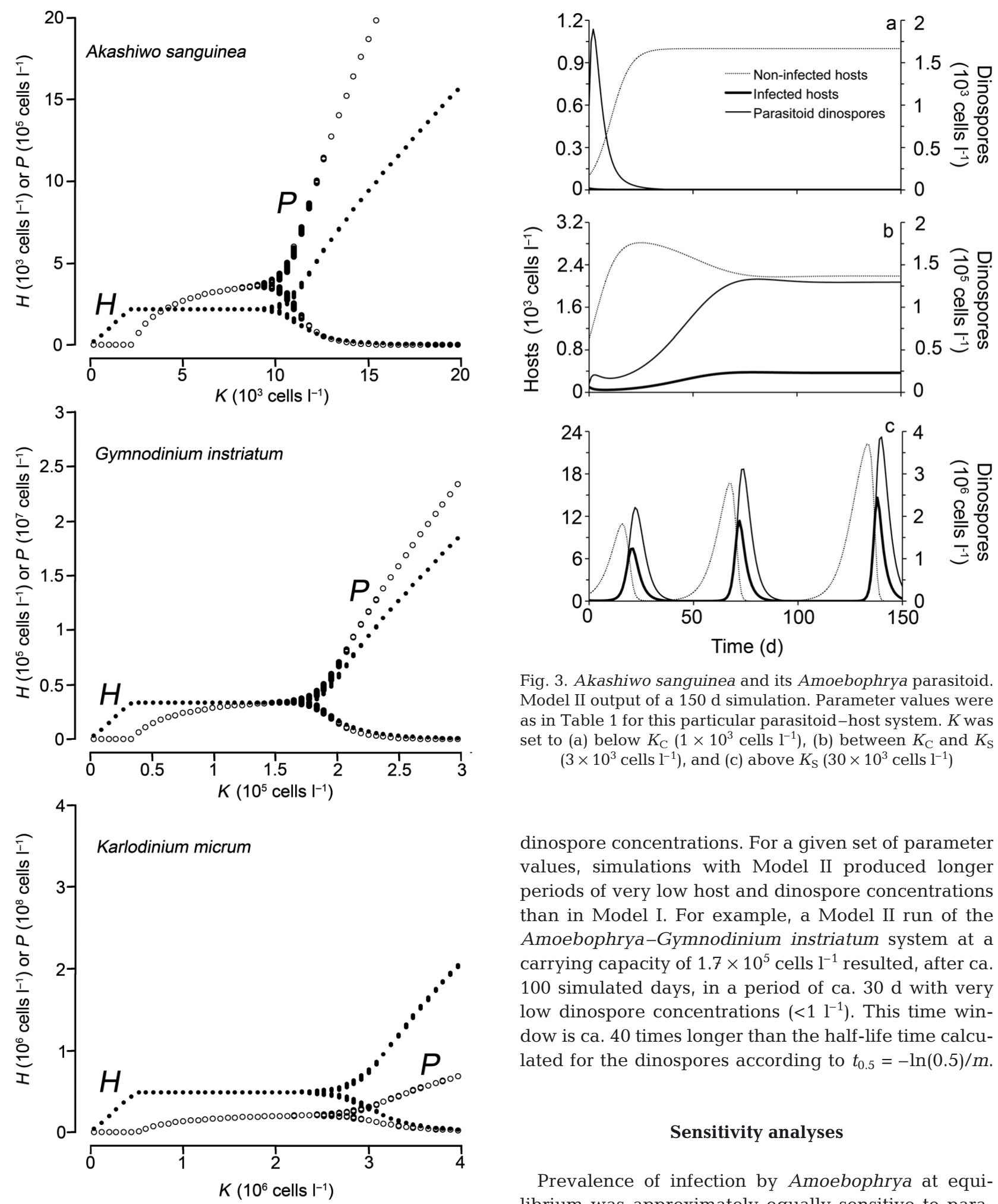

dinospore concentrations. For a given set of parameter values, simulations with Model II produced longer periods of very low host and dinospore concentrations than in Model I. For example, a Model II run of the Amoebophrya-Gymnodinium instriatum system at a carrying capacity of $1.7 \times 10^{5}$ cells $^{-1}$ resulted, after ca. 100 simulated days, in a period of ca. $30 \mathrm{~d}$ with very low dinospore concentrations $\left(<1 \mathrm{l}^{-1}\right)$. This time window is ca. 40 times longer than the half-life time calculated for the dinospores according to $t_{0.5}=-\ln (0.5) / \mathrm{m}$.

\section{Sensitivity analyses}

Fig. 2. Bifurcation analysis of Model II for the 3 Amoebophyra-dinoflagellate systems run with parameter values as in Table 1 at a range of carrying capacities $(K)$. Steady state concentrations, or maximum and minimum values in the case of unstable coexistence, of hosts $(H)$ and free-living parasites $(P)$ are plotted against $K$

Fig. 3. Akashiwo sanguinea and its Amoebophrya parasitoid. Model II output of a $150 \mathrm{~d}$ simulation. Parameter values were as in Table 1 for this particular parasitoid-host system. $K$ was set to (a) below $K_{\mathrm{C}}\left(1 \times 10^{3}\right.$ cells $\left.1^{-1}\right)$, (b) between $K_{\mathrm{C}}$ and $K_{\mathrm{S}}$ $\left(3 \times 10^{3}\right.$ cells $\left.l^{-1}\right)$, and (c) above $K_{\mathrm{S}}\left(30 \times 10^{3}\right.$ cells l$\left.^{-1}\right)$

Prevalence of infection by Amoebophrya at equilibrium was approximately equally sensitive to parameters $\varepsilon, a, r, h$, and $m$. Prevalence was negatively correlated with $m$, and positively correlated with variation in $\varepsilon, a, r$ and $h$. Within the range $-25 \%$ to $+25 \%$ of the original values, nearly linear relations were observed between each parameter and prevalence (Fig. 4). 


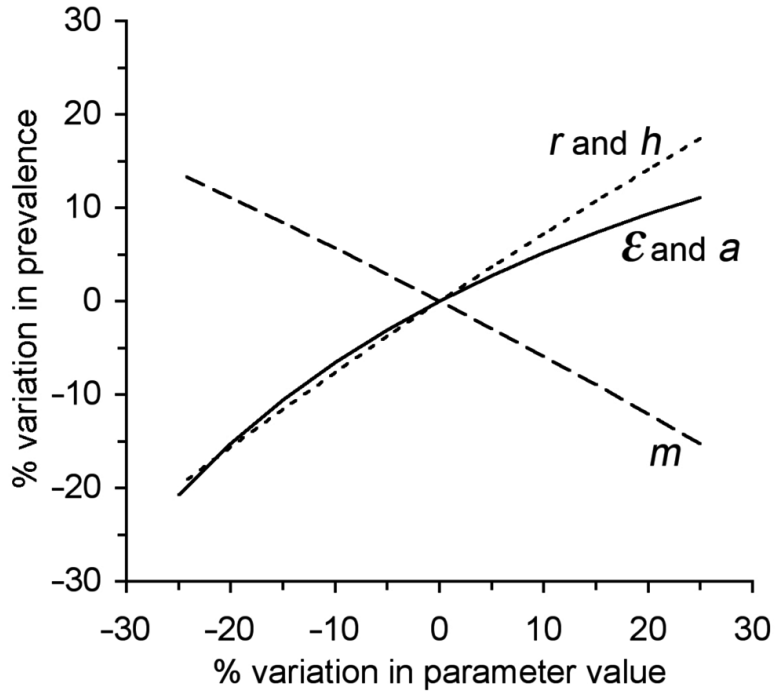

Fig. 4. Sensitivity analysis of Model II. Initial parameter values (corresponding to the position 0,0 in the diagram) were as in Table 1 for the Amoebophrya-Akashiwo sanguinea system.

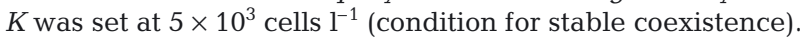
Each parameter was increased and decreased up to $25 \%$ (at $5 \%$ steps) of the initial values and plotted against the variation (in \% of the initial condition) in model output (parasite prevalence at equilibrium)

\section{DISCUSSION}

A classical predator-prey model (Model I) was the starting point of our Amoebophrya-dinoflagellate population dynamics model. To compare model results with field data, which are almost exclusively available as parasitoid prevalence, an infected host state variable (I) was introduced in Model II. This addition had a destabilizing effect similar to a previously described parasite-host model (May 1978). The introduction of infected hosts as a state variable may be a better representation of the parasites' natural behavior, since the time spent inside hosts (ca. 2 to $3 \mathrm{~d}$ ) is on the same order of magnitude as the time spent as free-living dinospores. One important advantage of this formulation (Model II) is that no extra parameters needed to be introduced. Besides having introduced an infected host variable to compare with field observations, there was no longer a direct relation between infection and production of new dinospores. This causes a delay in dinospore production in comparison with Model I, and is more consistent with the relatively long handling time of the parasite. A similar approach has been recently used in a food web model including this kind of parasitoid of dinoflagellates (Montagnes et al. 2008). The present host-parasitoid model approach includes fewer processes (e.g. the vermiform phase of Amoebophrya is not included) and non-linear dynamics compared to linear parasite-host relationship in the
Anderson-May model described by Montagnes et al. (2008). Parameter estimation in our model was similar to Montagnes et al. (2008) in most cases. One of the main differences is that we estimated search rate of the parasite for hosts assuming non-saturating conditions at the host and parasitiod dinospore concentrations used by Coats \& Park (2002) in their experiments. On the other hand, Montagnes et al. (2008) used a simple linear relationship to express transmission rates. The use of more complex, non-linear descriptions in our model is justified by the relatively good availability of data for parameterization. The fact that we used a nonlinear model makes it possible to reach stable steady state coexistence, whereas this is not possible in linear models of isolated host-parasite systems. The addition of grazers leads to stable coexistence in the simulations by Montagnes et al. (2008). Had we chosen a linear model, the model would not have reached stable coexistence. Linear relationships are rare in nature (e.g. metabolic processes or functional and numerical responses), so our formulation is probably more realistic without making the model too complex to analyze the dynamics e.g. as a function of carrying capacity.

\section{Model parameterization and sensitivity}

Parameterization was based on experimental data from 3 dinoflagellate-Amoebophrya systems. A critical point in the parameterization was the dinospore search rate (a). In the experiment from which the parameter was estimated, the host concentrations remained constant $\left(10^{6}\right.$ hosts $\left.\mathrm{l}^{-1}\right)$ and dinospore concentrations varied to reach different dinospore:host ratios (Coats \& Park 2002). In the present work, a host concentration of $10^{6}$ hosts $l^{-1}$ was assumed to be not yet saturating, i.e. infection rate is more strongly dependent on the dinospore search rate, and less on handling time (intracellular development time) of the parasitoid. If this assumption is false, then a was underestimated, but never overestimated. Furthermore, it was assumed that infectivity of surviving dinospores is constant with time. It seems, however, that the ability of dinospores to infect new host cells decreases as the dinospores age (Coats \& Park 2002), something that is not included in the model. One way to compensate for this, without changing the model structure, would be to increase the value of $m$.

The validation of the parameter search rate (a) and natural mortality $(m)$ was done using independent experimental data. However, the experiments used for validation were done with extremely synchronized cultures, which the continuous time model used in this study was not able to reproduce. This may explain the overestimation of prevalence for the host Akashiwo 
sanguinea at a low dinospore:host ratio, as well as the poor fit between model and real data for dinospore concentration, and is considered to be more a consequence of the model structure than parameterization. The relatively low prevalence for Karlodinium micrum at both low and high dinospore:host ratios could be explained by an underestimation of search rate for that parasitoid-host system.

Sensitivity analyses were done at a carrying capacity within the range of stable coexistence. Under these conditions, prevalence is not strongly dependent on a single parameter. The model may be regarded as relatively robust, since the relative change in output is in the same order of magnitude as the relative change of all parameters excluding $K$.

\section{Comparison between model output and experimental and field data}

The temporal dynamics of Amoebophrya epidemics (i.e. peaks of high prevalence) observed in some natural host populations is on the order of $4 \mathrm{wk}$ for Gonyaulax catenella (=Alexandrium catanella) (Nishitani et al. 1985), 2 to $4 \mathrm{wk}$ for Scrippsiella trochoidea and Gymnodinium sanguineum (= Akashiwo sanguinea) and 3 to $6 \mathrm{wk}$ for Gyrodinium uncatenum (Coats et al. 1996). The period of epidemics for A. sanguinea in our model is slightly longer (ca. $6 \mathrm{wk}$ ) compared to available field data for this parasitoid-host system.

In Model II, the total time spent at very low host and Amoebophrya dinospore concentrations is longer than in Model I, at comparable parameter values and carrying capacity. This would mean that following Model II, there is a higher risk of extinction due to stochastic effects for either the parasitoid or the host compared to Model I. The long periods with very low host concentrations predicted by Model II after an infection outbreak at high carrying capacity might represent a situation for the host where the risk of extinction is enhanced as compared to a situation with no parasitoid present. In fact, in some cases host populations never seem to recover from repeated parasitic outbreaks (Chambouvet et al. 2008, Montagnes et al. 2008). However, parasitoids might be more sensitive to extinction risk than hosts, because their survival is fully dependent on the interaction. Any process in addition to the internal dynamics of Model II that will decrease the concentration of dinospores or physically separate them from the host cells (dispersion, grazing, sedimentation) will decrease their probability of survival.

In general, Model II produced similar results to the laboratory experiments in Coats \& Park (2002) regarding the general patterns of non-infected and infected hosts, and dinospore concentrations. However, parasite-induced mortality in the experiments only started after the intracellular development time of Amoebophrya (1.4 to $2.4 \mathrm{~d})$ due to the fact that there were no infected hosts at $\mathrm{t}=0$. In our model, mortality starts earlier, sometimes directly at high dinospore:host ratios due to the fact that it is a continuous-time model, and the transition between the states is calculated as a probability, rather than a fixed time delay. This also caused dinospore concentrations in our simulations to develop faster than in the experimental observations. Natural populations are likely not as synchronized as laboratory cultures; therefore, the discrepancy between laboratory and model results is expected to be less important for the prediction of field population dynamics. Although other types of models may better describe the time-course in synchronized populations (e.g. individual-based models), the present model has the advantage of being simple with regard to computation time and possibilities for analytical solutions.

Our modeling exercise predicts that, at stable coexistence, the dinospore concentration will be ca. 50 to 100 times the concentration of host cells. As expected, this is in agreement with the experimental data from which the parameters were derived (Coats \& Park 2002). Our model would gain considerable strength if this finding could be verified with field data. Amoebophrya-like DNA sequences have regularly been observed in the picoplankton size class from different marine environments (Groisillier et al. 2006, Lin et al. 2006, Marie et al. 2006, Dolven et al. 2007) suggesting that they are widespread members of plankton communities. Quantification of Amoebophrya dinospores using FISH probes have shown concentrations up to $10 \times 10^{5} \mathrm{l}^{-1}$ in natural samples from waters with infected dinoflagellate hosts (Chambouvet et al. 2008). In this case, dinospores were only ca. 2 times more abundant than hosts, much lower than our model prediction at steady state. The host species investigated by Chambouvet et al. (2008) did not include the ones used in the present work to parameterize the model. It is possible that the dynamics of other Amoebophrya-dinoflagellate systems differ so that fewer dinospores are needed to maintain infection (e.g. a parasitoid with very low $m$ ). To our knowledge, other data on dinospore concentration in field samples with infected host populations are not available.

Natural populations of Amoebophrya and its hosts will be subject to more interactions and more varying conditions than are included in our model. For example, dinospore mortality can be expected to be higher in natural populations due to microzooplankton grazing (Johansson \& Coats 2002). Grazing by heterotrophic flagellates on Amoebophrya dinospores was included in the food web model made by Montagnes et 
al. (2008) as one of the loss factors for the parasite. Spatial variation in natural situations, due to transport and imperfect mixing (patchiness), which subsequently cause large variation in host cell and dinospore concentrations at small spatial scales, may influence encounter rates of dinospores and the risk for predation of dinospores. Furthermore, features related to the biology of the organisms, e.g. resting cysts of the parasite, multiple infection of the host, and formation of parasite-resistant resting cysts of the host, are also relevant for the dynamics and can be dealt with in later, more complex models.

In principle, the model described here gives reliable estimates over a wide range of host and parasite concentrations. However, model validation at low host or parasite concentrations is limited by the detection limits of the organisms in natural waters or experimental set ups. Without incorporation of the influence of the factors described above into the model, the model has the potential to give good estimates for up to a few weeks, depending on the dynamics of each particular environment. Whether steady-state dynamics (i.e. conditions for stable coexistence) will be reached in nature relies on host carrying capacity and the dynamics of the environment. Montagnes et al. (2008), for example, chose a time frame of $50 \mathrm{~d}$ for their model simulations, because pelagic environments are likely to suffer significant perturbations beyond this time. Once again, in more stable environments with $K_{\mathrm{C}}<K<$ $K_{\mathrm{S}}$, stable coexistence is more likely to occur than in other situations.

Amoebophrya dinospores kept in culture have relatively high mortality rates (Coats \& Park 2002). During winter periods, carrying capacities are expected to be well below that for stable coexistence. This represents a high risk of extinction for the parasitoid in temperate waters. Even though in our simulation we could get overwintering populations that were still able to develop stable populations in the next season, the probability for this will be very dependent on stochastic processes influencing the survival of dinospores. Anderson \& May (1981) proposed 3 possible mechanisms for parasites to cope with wide fluctuations in host abundance: (1) vertical transmission, (2) occult infection, and (3) long-lived free-living infective stages. For Amoebophrya, vertical transmission seems unlikely, at least from what is known from experimental systems. Long-lived, free-living infective stages do not seem to exist, also based on experimental studies. Dormant cells or cysts have so far not been reported. We hypothesize that occult infection, or infection of an alternative host, is the strategy for Amoebophrya to facilitate survival during winter. Several cultured Amoebophrya-dinoflagellate systems are highly hostspecific, although exceptions have been documented
(Coats et al. 1996, Sengco et al. 2003, Park et al. 2004, Kim 2006, Leblond et al. 2006). An ability to infect and successfully reproduce using alternative hosts will change the system's dynamics, giving the parasitoid a higher probability of survival at long periods of low primary host densities.

\section{Using the model to predict control of host populations by the parasitoid}

The conditions under which the host population is under control of its parasitoid can be regarded as an operational window for the potential use of the parasite to biologically control an outbreak of the host. Regarding carrying capacity, which may indicate trophic state of the environment, this operational window (i.e. the range $K_{\mathrm{S}}-K_{\mathrm{C}}$ ) was considerably reduced by introducing Model II as compared with Model I. The hypothesis that stable control of host biomass concentration only occurs within this window is testable. The choice of model could therefore influence the cost-effectiveness calculations in case this kind of parasite is used as a biological control mechanism against harmful algal blooms.

Suggestions have been made to use Amoebophrya as a biocontrol agent for harmful dinoflagellate blooms (Taylor 1968, Coats et al. 1996, Anderson 1997). Infections by Amoebophrya might be as or more important than grazing in controlling dinoflagellate blooms in nature (Chambouvet et al. 2008, Montagnes et al. 2008). To estimate the feasibility of biocontrol measures or the effectiveness of Amoebophrya in controlling natural host populations, model predictions are essential. The current model is very simple from a mathematical point of view, making it in principle applicable under many different conditions. The model provides the possibility to explore the dynamical behavior of the system beyond time scales that are practically feasible during laboratory experiments. It can be used as it is in closed systems (e.g. mesocosm experimental set ups, embayment with low water exchange) or easily incorporated into larger model systems.

Acknowledgements. We thank D. W. Coats (SERC, MD, USA) for sharing raw data from previously published experimental work with Amoebophrya, T. Petzholdt (Technical University of Dresden, Germany) for help with adapting the R scripts, G. O. Fistarol for critical reading of the first versions of the text, and D. J. S. Montagnes and 2 anonymous reviewers for constructive comments on the manuscript.

\section{LITERATURE CITED}

Anderson DM (1997) Turning back the harmful red tide. Nature 388:513-514 
Anderson RM, May RM (1980) Infectious diseases and population cycles of forest insects. Science 210:658-661

Anderson RM, May RM (1981) The population dynamics of microparasites and their invertebrate hosts. Philos Trans R Soc Lond B Biol Sci 291:451-524

Cachon J, Cachon M (1987) Parasitic dinoflagellates. In: Taylor FJR (ed) The biology of dinoflagellates. Blackwell Scientific Publications, Oxford, p 571-610

Chambouvet A, Morin P, Marie D, Guillou L (2008) Control of toxic marine dinoflagellate blooms by serial parasitic killers. Science 322:1254-1257

- Coats DW, Bockstahler KR (1994) Occurrence of the parasitic dinoflagellate Amoebophrya ceratii in Chesapeake Bay populations of Gymnodinium sanguineum. J Eukaryot Microbiol 41:586-593

> Coats DW, Park MG (2002) Parasitism of photosynthetic dinoflagellates by three strains of Amoebophrya (Dinophyta): parasite survival, infectivity, generation time, and host specificity. J Phycol 38:520-528

> Coats DW, Adam EJ, Gallegos CL, Hedrick S (1996) Parasitism of photosynthetic dinoflagellates in a shallow subestuary of Chesapeake Bay, USA. Aquat Microb Ecol 11:1-9

Dolven JK, Lindqvist C, Albert VA, Bjorklund KR, Yuasa T, Takahashi O, Mayama S (2007) Molecular diversity of alveolates associated with neritic North Atlantic radiolarians. Protist 158:65-76

Groisillier A, Massana R, Valentin K, Vaulot D, Guillou L (2006) Genetic diversity and habitats of two enigmatic marine alveolate lineages. Aquat Microb Ecol 42:277-291

Gunderson JH, Goss SH, Coats DW (1999) The phylogenetic position of Amoebophrya sp. infecting Gymnodinium sanguineum. J Eukaryot Microbiol 46:194-197

Hallegraeff GM (2003) Harmful algal blooms: a global overview. In: Hallegraeff GM, Anderson DM, Cembella AD (eds) Manual on harmful marine microalgae. UNESCO, Paris, p 25-49

Janson S, Gisselson LÅ, Salomon PS, Granéli E (2000) Evidence for multiple species within the endoparasitic dinoflagellate Amoebophrya ceratii as based on 18S rRNA gene-sequence analysis. Parasitol Res 86:929-933

Johansson M, Coats DW (2002) Ciliate grazing on the parasite Amoebophrya sp. decreases infection of the red-tide dinoflagellate Akashiwo sanguinea. Aquat Microb Ecol 28: $69-78$

Kim S (2006) Patterns in host range for two strains of Amoebophrya (Dinophyta) infecting thecate dinoflagellates: Amoebophyra spp. ex Alexandrium affine and ex Gonyaulax polygramma. J Phycol 42:1170-1173

Kim S, Park MG, Yih W, Coats DW (2004) Infection of the bloom-forming thecate dinoflagellates Alexandrium affine and Gonyaulax spinifera by two strains of Amoebophrya (Dinophyta). J Phycol 40:815-822

Kim S, Park MG, Kim KY, Kim CH, Yih W, Park JS, Coats DW (2008) Genetic diversity of parasitic dinoflagellates in the genus Amoebophrya and its relationship to parasite biology and biogeography. J Eukaryot Microbiol 55:1-8

Editorial responsibility: Hans Heinrich Janssen, Oldendorf/Luhe, Germany
Leblond JD, Sengco MR, Sickman JO, Dahmen JL, Anderson DM (2006) Sterols of the syndinian dinoflagellate Amoebophrya sp., a parasite of the dinoflagellate Alexandrium tamarense (Dinophyceae). J Eukaryot Microbiol 53:211-216

> Lin S, Zhang H, Hou Y, Miranda L, Bhattacharya D (2006) Development of a dinoflagellate-oriented PCR primer set leads to detection of picoplanktonic dinoflagellates from Long Island Sound. Appl Environ Microbiol 72:5626-5630

Maranda L (2001) Infection of Prorocentrum minimum (Dinophyceae) by the parasite Amoebophrya sp. (Dinoflagellea). J Phycol 37:245-248

Marie D, Zhu F, Balague V, Ras J, Vaulot D (2006) Eukaryotic picoplankton communities of the Mediterranean Sea in summer assessed by molecular approaches (DGGE, TTGE, QPCR). FEMS Microbiol Ecol 55:403-415

> May RM (1978) Host-parasitoid systems in patchy environments: a phenomenological model. J Anim Ecol 47:833-844

May RM, Anderson RM (1978) Regulation and stability of host/parasite population interactions. II. Destabilizing processes. J Animal Ecol 47:249-267

> Montagnes DJS, Chambouvet A, Guillou L, Fenton A (2008) Responsibility of microzooplankton and parasite pressure for the demise of toxic dinoflagellate blooms. Aquat Microb Ecol 53:211-225

Nishitani L, Erickson G, Chew KK (1985) Role of the parasitic dinoflagellate Amoebophrya ceratii in control of Gonyaulax catenella populations. In: Anderson DM, White AW, Baden DG (eds) Toxic dinoflagellates. Elsevier Science, New York, NY, p 225-230

> Park MG, Yih W, Coats DW (2004) Parasites and phytoplankton, with special emphasis on dinoflagellate infections. J Eukaryot Microbiol 51:145-155

Rosenzweig ML (1971) Paradox of enrichment: destabilization of exploitation ecosystems in ecological time. Science 171: 385-387

Salomon PS, Janson S, Granéli E (2003a) Parasitism of Dinophysis norvegica by Amoebophrya sp. in the Baltic Sea. Aquat Microb Ecol 33:163-172

Salomon PS, Janson S, Granéli E (2003b) Multiple species of the dinophagous dinoflagellate genus Amoebophrya infect the same host species. Environ Microbiol 5:1046-1052

Salomon PS, Janson S, Gisselson LÅ, Graneli E (2006) Analytical detection of parasite infection of Dinophysis norvegica using FISH probes. Afr J Mar Sci 28:353-355

> Salomon PS, Granéli E, Neves MHCB, Rodriguez EG (2009) Infection by Amoebophrya spp. parasitoids of dinoflagellates in a tropical marine coastal area. Aquat Microb Ecol 55:143-153

Sengco MR, Coats DW, Popendorf KJ, Erdner DL, Gribble KE, Anderson DM (2003) Biological and phylogenetic characterization of Amoebophrya sp. ex Alexandrium tamarense. Second Symposium on Harmful Marine Algae in the US, Dec 8-13, 2003, Woods Hole, Abstract 57, p 46

Taylor FJR (1968) Parasitism of the toxin-producing dinoflagellate Gonyaulax catenella by the endoparasitic dinoflagellate Amoebophrya ceratii. J Fish Res Board Can 25: 2241-2245

Submitted: August 12, 2009; Accepted: September 23, 2010

Proofs received from author(s): November 3, 2010 